\title{
Low Power Sensor Systems Technologies for Environmental Air-Monitoring
}

\author{
S. H. Brongersma \\ Holst Centre/imec, High Tech Campus 31, 5656AE Eindhoven, The Netherlands
}

\begin{abstract}
Distributed monitoring of air quality in the environment requires ultra-low power components for autonomous systems. These include the actual transducer, an analog interface, some local processing capability and a wireless connection. To power the system one needs a battery or energy harvester in combination with smart power management. The approximate target is an overall system power of 100 microWatt, which leaves only a fraction of that for (bio-) chemical sensing.

Although a wide range of sensor technologies is available, power consumption of existing technologies is typically high because they require light (e.g. IR for CO2), heating (metal oxides), etc. Consequently, novel sensing technologies are required that work around these limitations.

Recent developments in this direction include a mems-based electronic nose for volatile sensing that uses piezo-electrics instead of optics for resonance frequency monitoring; metal oxide $\mathrm{CO} 2 / \mathrm{O} 2$ sensors that operate at significantly lower temperatures (or even room temperature); low power $\mathrm{NO}_{\mathrm{x}}$ sensors that operate in the sub-ppb range.

For a few chosen technologies, the sensors are combined with read-out and driver electronics to then implement them in system demonstrators. These are a combination of off-the-shelf components and inhouse developments on read-out, wireless and platform approaches.
\end{abstract}

Keywords: Sensors arrays; Low power sensor systems; bio-chemical sensors; Air quality; 